\title{
How should abridged scientific articles be presented in journals? A survey of readers and authors
}

\author{
Marcus Müllner, Fabian Waechter, Sara Schroter, Barbara Squire
}

\section{Abstract}

SeVeral SCIENTIFIC and General medical JOURNAls publish fulllength articles on their Web sites and abridged versions in their print journals. We surveyed a stratified random sample of BMJ readers and authors to elicit their preferred format for the abridged print version. Each participant received a research paper abridged in 3 different formats: conventional abridged version, journalistic version and enhanced-abstract version. Overall, $45 \%$ (95\% confidence interval [Cl] 42\%-48\%) of the respondents said they liked the conventional version most, $31 \%(95 \% \mathrm{Cl} 28 \%-34 \%)$ preferred the journalistic version and $25 \%(95 \% \mathrm{Cl} 22 \%-27 \%)$ preferred the enhanced-abstract version. Twenty-eight percent $(95 \% \mathrm{Cl} 25 \%-32 \%)$ indicated that use of the journalistic format for abridged articles would very likely stop them from submitting papers to BMJ, and 13\% (95\% $\mathrm{Cl} 11 \%-16 \%$ ) said the use of the enhanced-abstract version would stop them from submitting to BMJ. Publishers of general medical journals who publish shortened articles should consider that authors and readers prefer a more conventional style of abridged papers.

CMAJ 2005;172(2):203-5

$\mathrm{T}$ o allow critical appraisal of a scientific paper, a certain amount of space is needed to describe the methods and results. Editorial space is scarce, however, and editors struggle to meet the expectations of both authors and readers. Moreover, it seems that many readers read only parts of scientific papers. Now that electronic publishing is available, several scientific and general medical journals have adopted strategies to publish detailed articles on their Web sites, often in advance of the print journal publication, with a more concise presentation appearing in the print journal.

Since 2000 most papers in $B M \mathcal{F}$ have been published in their full-length form on the journal's Web site and in an abridged form in the print journal. ${ }^{1}$ Currently the abridgement is simply a condensed version of the full-length paper (about 30\%-50\% shorter). The conventional structure of scientific papers is maintained, and the original wording is hardly altered.
There are many alternative forms of presenting abridged scientific information. "Serious" magazines such as The Economist use a journalistic approach, presenting the salient information at the beginning and more technical details later in the text. Medical journals such as EvidenceBased Medicine use enhanced abstracts, in which details of the results are presented (including a table or figure), and the main text - if there is any - provides context and interpretation.

We performed a survey to find which format of short version - the conventional approach, a journalistic version or a version with an enhanced abstract - of scientific articles is preferred by readers and authors. We randomly sampled 2 papers each of 6 study designs (randomized controlled trial, systematic review, meta-analysis, cohort study, case-control study and qualitative study) published in conventional abridged form ${ }^{2,3}$ in $B M 7$ between January 2000 and June 2002. The conventional format is a condensed version of the full-length paper and contains 1300-1500 words; the structure of the paper (Introduction, Methods, Results, Discussion) is retained. ${ }^{3}$ For each paper we prepared 2 additional abridged formats: a journalistic version ${ }^{4}$ and an enhanced-abstract version (see the online appendix at www.cmaj.ca/cgi/content/full/172/2/203/DC1 for a sample of 3 abridgements of 1 paper). The journalistic version was based on a style typically used by "serious" newspapers, with the following subheadings: "Why we carried out this study," "The background," "What were the main findings?," "How did we perform the study?" and "Why are these results important?" This format had no abstract, the paper starting instead with the main findings and conclusions in the first paragraph, followed by less salient details. The enhanced-abstract version provided detailed results in the abstract (including a table or figure), and the main body of the text was explanatory, focusing on the context and discussion.

We drew a stratified random sample from $1728 \mathrm{UK}$ and non-UK readers and 360 corresponding authors of a research paper published in $B M 7$ between January 2000 and June 2002. Each participant received all 3 abridged versions of 1 of 12 research papers. The study design of the paper 
and the order of versions were allocated randomly to each participant.

We asked the respondents to rank the 3 versions in order of preference and to indicate how strongly they felt about their preference. We also asked participants whether use of either of the journalistic or enhanced-abstract formats instead of the conventional format would prevent them from submitting papers to $B M F$ in the future. We offered a book voucher as an incentive and sent 2 reminders to nonrespondents. ${ }^{4}$

Of the sample of 2088 respondents, 55 were excluded (43 had incorrect mailing addresses, and 12, being both readers and authors, were selected more than once because of overlap in the databases). Of the 2033 eligible participants, 1002 (49\%) responded. The response rate was higher for authors (220/321 [68\%]) than readers $(782 / 1712$ [46\%]). Item nonresponse was minimal.

A total of $45 \%$ (95\% confidence interval [CI] $42 \%-48 \%$ ) of the respondents (446/997) (42\% of readers and $56 \%$ of authors) said that they liked the conventional abridged version most (Fig. 1). The next most preferred format was the journalistic version $(31 \%$ [95\% CI
28\%-34\%] [306/997]), followed by the enhanced-abstract version (25\% [95\% CI 22\%-27\%] [245/997]). When we stratified for location (UK v. non-UK) and audience (reader v. author), the conventional abridged version remained the preferred version, except among UK readers, who gave the 3 versions similar rankings (Fig. 1).

Most respondents (87\% [95\% CI 85\%-89\%] [863/994]) liked their first choice "a lot." Thirteen percent $(95 \%$ CI $11 \%-15 \%)(127 / 994)$ liked their first choice only "a little," and none said they "don't like it at all." Four respondents $(0.4 \%)$ did not have an opinion.

A total of 268 respondents said they were unlikely to submit a paper to $B M 7$ in the future. Of the remaining 734 respondents, $28 \%$ (95\% CI 25\%-32\%) indicated that use of the journalistic format for abridgement would very likely stop them from submitting papers to BMF, and 13\% (95\% CI $11 \%-16 \%$ ) said that use of the enhanced-abstract format as abridgement would.

Our findings that a conventional abridged version was preferred by $B M 7$ authors and readers and that many respondents indicated that use of journalistic and enhancedabstract formats would prevent them from submitting fu-

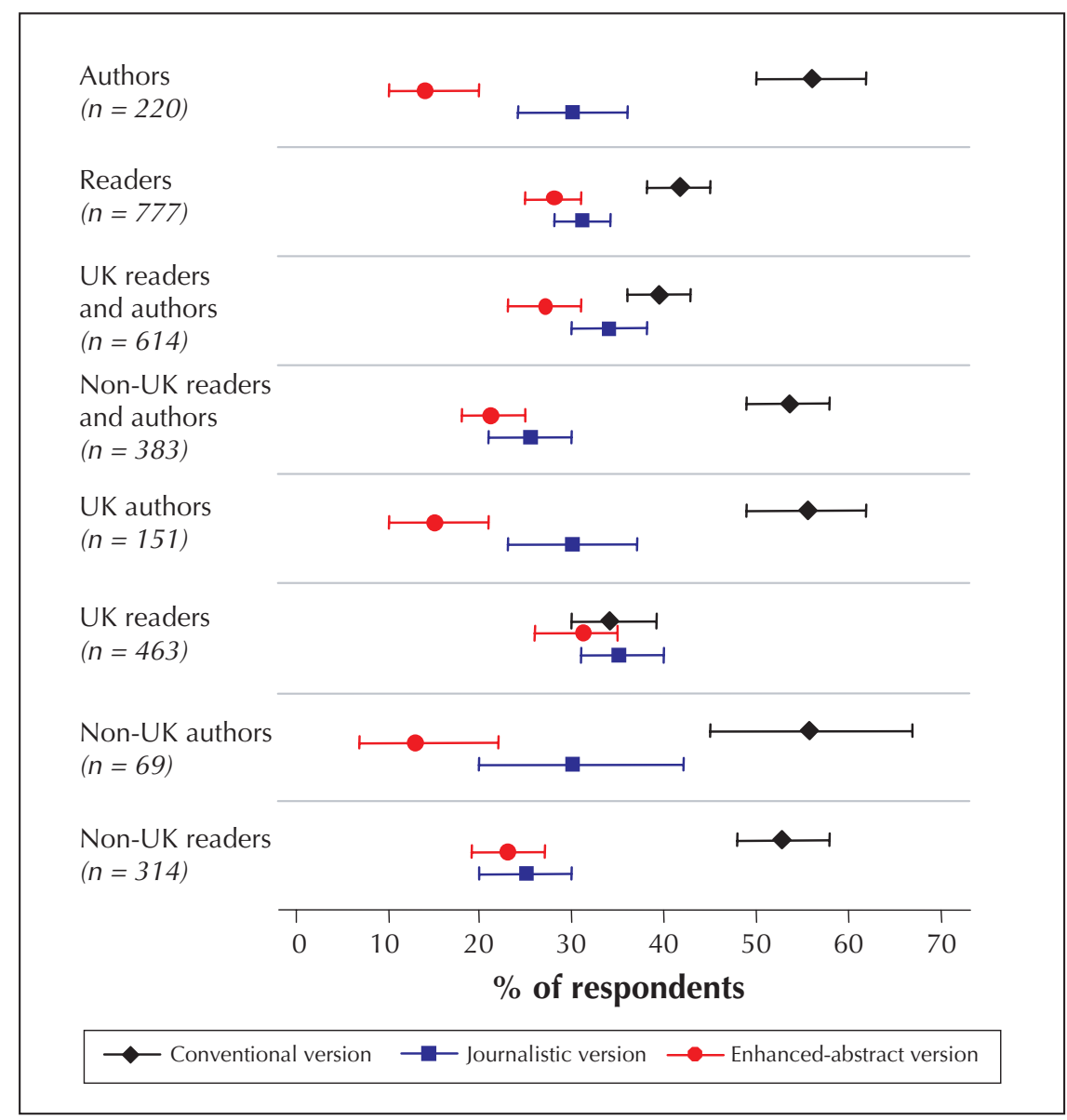

Fig. 1: Type of short version of scientific article most preferred by $B M J$ authors and readers, stratified for audience (authors $v$. readers) and geographic location (UK v. non-UK). Error bars represent the 95\% confidence interval. 
ture papers to the journal are important signals for editors. They indicate that contributors' opinions should be considered when planning major changes for the presentational style of scientific papers.

The main limitation of our study is the low response rate for readers (46\%), despite strategies to optimize response rates. ${ }^{4}$ However, we believe that the information we received from readers is still useful, since we have no reason to believe that any particular selection mechanism is active other than lack of interest in the presentation of research papers.

Publishers of scientific and general medical journals who use or are considering using abridgements of scientific articles should bear in mind that a conventional format of abridged papers may be the choice most likely to satisfy both readers and authors.

This article has been peer reviewed.

From the Medizinische Universität Wien, Austria (Müllner), and BMF, British Medical Association, London, UK (Müllner, Waechter, Schroter, Squire)

Competing interests: Marcus Müllner works as an associate editor with BMF and was involved in the conception, birth and further development of the electroniclong, paper-short model. At the time the research was conducted, Fabian
Waechter was employed by the $B M 7$ to conduct this and other research projects related to medical publishing. Sara Schroter is employed by the $B M 7$ to conduct research into editorial processes such as peer review.

Contributors: All authors contributed substantially to study conception and design and to data interpretation. Fabian Waechter and Sara Schroter collected the data. Marcus Müllner performed the data analysis and wrote the first draft. All authors gave final approval of the submitted version to be published.

\section{References}

1. Delamothe T, Müllner M, Smith R. Pleasing both authors and readers [editorial]. BMF 1999;318:888-9.

2. Müllner M, Groves T. Making research papers in the $B M 7$ more accessible [editorial]. BM7 2002;325:456.

3. Müllner M. Publishing short articles in the print journal and full articles on the Web? The BMF is doing it with most research papers. Eur 7 Sci Edit 2003; 29:6-9.

4. Edwards P, Roberts I, Clarke M, DiGuiseppi C, Pratap S, Wentz R, et al. Increasing response rates to postal questionnaires: systematic review. BM7 2002 324:1183.

Correspondence to: Dr. Marcus Müllner, Univ. Klinik für Notfallmedizin, Allgemeines Krankenhaus Wien, Währinger Gürtel 18-20 / 6D, A-1090, Austria; fax +41 140400 2512; marcus.muellner@meduniwien.ac.at

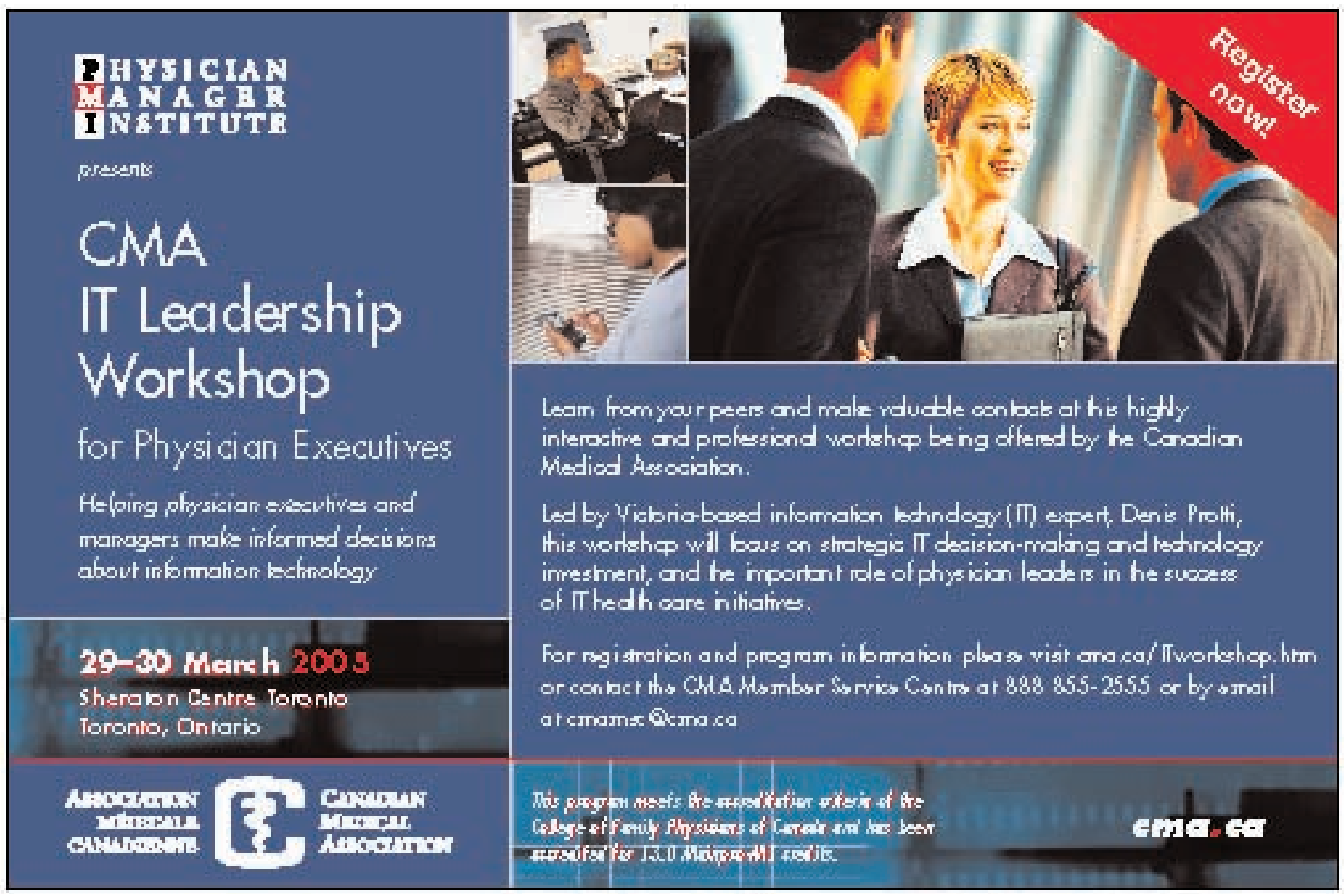

